\title{
MOOD DISORDERS
}

\section{Balancing anxiety and social desire}

\author{
Persistent negative emotional states, such as anxiety, suppress social behavior and vice versa. A new report \\ identifies a novel neural circuit that generates persistent anxiety states and describes how competing excitatory \\ and inhibitory components of this circuit battle to pattern social behavior.
}

\section{Dakota Blackman and Annegret L. Falkner}

$\mathrm{M}$ any social interactions are rewarding, perhaps even soothing. Imagine, for example, a cuddle with a loved one on the couch after a hard day or the feeling of a soundly sleeping baby on one's chest. However, other social encounters, like discovering an unwelcome intruder in your house, may be stressful, perhaps even able to trigger actions linked to self-preservation, like aggression or escape. Moreover, the stressful emotions evoked by these encounters may linger for hours or days beyond the encounter itself, creating lasting mental and behavioral disruption.

This type of sustained fear, commonly referred to as 'anxiety', has long been appreciated to be intricately intertwined with the execution of social behaviors. Anxiety and stress have been shown to reduce levels of parental care in many species $^{1}$, and common forms of anxiety, including social anxiety and parental anxiety, may be triggered by the social interactions themselves. However, the reverse is also true: social contact can reduce the long-term negative consequences of stress, an effect termed 'social buffering'? Though it is clear that these negative emotions and social behaviors can affect each other, how these processes inform each other at the neural level is still a matter of debate. In this issue of Nature Neuroscience, Zhang and colleagues identify a novel subcortical circuit that can potentially mediate the push and pull between positive social behavior and negative affective state ${ }^{3}$.

The neural mechanisms underlying anxiety are known to be brain-wide phenomena ${ }^{4}$, and recent circuit level descriptions have implicated distributed networks in the amygdala, bed nucleus of the stria terminalis (BNST), hippocampus, and prefrontal cortex ${ }^{5}$. The hypothalamus has not traditionally been included in this network; instead, hypothalamic subregions such as the medial preoptic area (MPOA) have well-known roles in the generation and maintenance of specific social behaviors. In particular, the MPOA has a critical and well-established role in the generation and maintenance of several 'positive' social

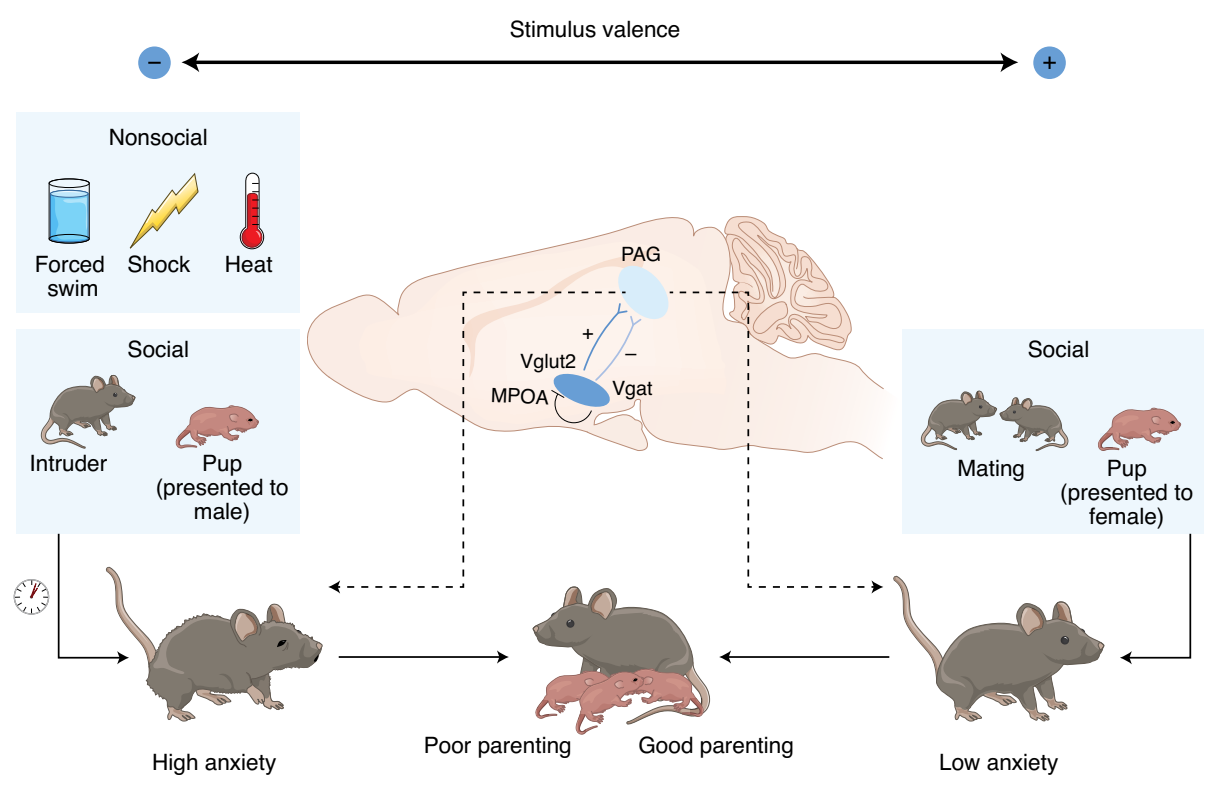

Fig. 1 | Distinct MPOA subpopulations differentially regulate anxiety and social behavior. Zhang et al. exposed mice to a variety of social and non-social stressors, determining that the excitatory (Vglut2) population in the hypothalamic MPOA is generally responsive to negative stimuli, long after the presentation of a stressor (indicated here by the clock, left). These neurons, along with the inhibitory (Vgat) population, project to downstream areas to increase and decrease anxiety behaviors, respectively, and influence parental behavior. PAG, periaqueductal gray.

behaviors, including parental behavior, sexual behavior, and social preference ${ }^{6-8}$. The neural populations that mediate these social behaviors are largely GABAergic, the molecularly complex majority population in the MPOA?

Zhang and colleagues instead turned their focus to the underexplored population of glutamatergic neurons in the MPOA $\left(\mathrm{MPOA}^{\mathrm{Vglut} 2}\right)$ and discovered a surprising role for this population in encoding 'negative' social and nonsocial experiences. To do this, the authors exposed mice to a variety of stressful conditions-including forced swimming, repeated inescapable shocks, and heat-and recorded neural activity at the single-neuron and population level. They found that in addition to activating more traditional anxiety network nodes, the stressors also activated this excitatory MPOA population. Importantly, activation of these neurons, as well as the anxious behaviors provoked by the stimuli, could persist for hours beyond the stressor. In addition to this sustained activation profile following stress, neurons generally did not discriminate between different stressors, indicating that this population encoded a generalized, persistent signal of a stressful experience.

In addition, Zhang et al. showed that this population not only responds to anxiety-promoting stimuli, it can bidirectionally control the expression of anxiety-related behaviors. Optogenetic activation of $\mathrm{MPOA}^{\text {vglut2 }}$ neurons increased pupil dilation and robustly enhanced anxiety-like behaviors in standard behavioral assays. Notably, mice worked hard to avoid acute stimulation of this 
neural population: given a choice between a stressor such as a swimming and acute stimulation of $\mathrm{MPOA}^{\text {Vglut2 } 2}$ neurons, the animals chose to jump into the water to avoid stimulation. In contrast, suppression of this population reduced behavioral readouts of anxiety. Importantly, silencing the neurons either during a stressful encounter or long after the encounter was over had similar anxiolytic effects. These findings demonstrate that the combination of both local and maintained representation of stress in the highly anxiogenic MPOA ${ }^{\text {vglut2 }}$ population is essential for generating anxiety-related behaviors.

Beyond encoding information about generalized non-social anxiogenic stimuli and experiences, this circuit node may have an additional role in socially-induced anxiety. The authors showed that social stimuli can themselves be stressors, with animals displaying heightened activation of the MPOA ${ }^{\text {vglut2 }}$ population in response to social stressors in a sexually dimorphic manner. Virgin males showed strong activation in response to encounters with another male or with pups, indicating that these encounters are aversive, and performed 'negative' social behaviors including attack. However, virgin females, for whom pups are presumably not aversive, showed no such behavioral responses to pups and did not demonstrate the same heightened MPOA ${ }^{\text {vlut2 }}$ activity when confronted with them. Suppression of the MPOA glutamatergic population during these social interactions also produced sexually dimorphic effects: it acutely reduced both inter-male and pup-directed aggression in males, and it actually increased the level of parental care in females. Importantly, these effects point to the circuit's ability to modify an ongoing spectrum of social behaviors, where particular behaviors, like aggression, may be associated with a high-anxiety state, while other behaviors, like parenting, may only be possible in a low-anxiety state (Fig. 1).

Lastly, the authors explored the role of this highly anxiogenic neural population within the wider social behavior circuit. The MPOA glutamatergic neurons receive direct local inhibition from MPOA GABAergic neurons, which respond robustly to positively valenced social stimuli. Both populations project to the periaqueductal gray, a midbrain structure with an established role in patterning social actions, including parental behavior and aggression ${ }^{7,10}$. The authors showed that activation of these GABAergic and glutamatergic populations respectively suppress and promote anxiety-related behaviors, which can affect the quality of parental care. This demonstrates that these MPOA subpopulations work in concert, playing opposing but intertwined roles to pattern social actions in the face of stress.

What could be the adaptive relevance of such a circuit? One possibility is that it enables individuals to directly compare environmental context to the value of a potential social interaction on a moment-to-moment basis. For example, if anxiogenic MPOA neurons indicate that an individual feels unsafe, this may be a time to prioritize personal safety over socially pleasurable behaviors, like mating. However, it is clear from these data how this comparison can go awry: over-activation of this population can lead to behaviors that are highly maladaptive for survival, like poor parenting. To counteract this, the MPOA GABAergic population provides both a potential 'brake' on anxiety both at the local circuit level and, through a competing projection to downstream behavioral actuators, allowing for 'positive' social behaviors to actively suppress negative affective state. More research needs to be done to discover how this circuit might weigh competing stimuli of opposite valence to potentially mediate social buffering or to make active choices about whether to parent or attack under stress. This may occur as a local 'winner-take-all' or may require the activation of the integrated brain-wide anxiety circuit. In particular, a parallel circuit in the bed nucleus of the stria terminalis (BNST) appears to perform a similar function through competing excitatory and inhibitory projections to midbrain dopaminergic neurons ${ }^{11}$, although how these networks inform each other under stress is unknown. While these data provide a thorough investigation of anxiogenic properties of the MPOA, we still lack insight about the computations being performed by this circuit during natural behavior and to its role within the larger anxiety network.

The findings also raise interesting questions about the neural coding of emotion. One of the hallmarks of emotions is that, in addition to positive and negative valence, they have persistence ${ }^{12}$. As anyone who has ever felt a sudden burst of rage or fear can attest to, these negative emotions often have a quick rise but a slow decay. The MPOA glutamatergic neurons provide a potential neural substrate for mediating this persistence, though the mechanism underlying the ability of this population to encode this persistent 'stress memory' has yet to be elucidated. This paper thus provides an exciting entry point for mechanistic dissection of emotional persistence.

Lastly, given the robustness of the anxiolytic properties of this circuit, this paper points to a promising clinical target for social anxiety. Globally, approximately 273 million people suffer from anxiety disorders, and these numbers have only increased in the face of the coronavirus pandemic, with those who already suffer from such disorders at greater risk for further negative mental health consequences ${ }^{13}$. To many, social interactions with others now act as acute stressors: a distant cough on a train or a mask-less fellow shopper at the grocery store can elicit fear and anxiety that may not have previously been present. In normal circumstances, after long bouts of social isolation, we crave social interaction ${ }^{14}$. However, in the midst of lockdowns and social distancing, our need to understand how new anxieties may alter social choices underscores the importance of translational applications of this paper's findings.

\section{Dakota Blackman and} Annegret L. Falkner (D) $₫$

Princeton Neuroscience Institute, Princeton, NJ, USA.

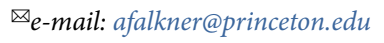

Published online: 5 March 2021 https://doi.org/10.1038/s41593-021-00812-w

References

1. Pawluski, J. L., Lonstein, J. S. \& Fleming, A. S. Trends Neurosci. 40, 106-120 (2017).

2. Kikusui, T., Winslow, J. T. \& Mori, Y. Phil. Trans. R. Soc. Lond. B 361, 2215-2228 (2006)

3. Zhang, G.-W. et al. Nat. Neurosci. https://doi.org/10.1038/s41593020-00784-3 (2021).

4. Papez, J. W. J. Neuropsychiatry Clin. Neurosci. 7, 103-112 (1995).

5. Adhikari, A. Front. Behav. Neurosci. 8, 112 (2014)

6. McHenry, J. A. et al. Nat. Neurosci. 20, 449-458 (2017).

7. Kohl, J. et al. Nature 556, 326-331 (2018).

8. Fang, Y.-Y., Yamaguchi, T., Song, S. C., Tritsch, N. X. \& Lin, D. Neuron 98, 192-207.e10 (2018).

9. Moffitt, J. R. et al. Science 362, eaau5324 (2018).

10. Falkner, A. L. et al. Neuron 106, 637-648.e6 (2020).

11. Jennings, J. H. et al. Nature 496, 224-228 (2013).

12. Anderson, D. J. \& Adolphs, R. Cell 157, 187-200 (2014).

13. Xiong, J. et al. J. Affect. Disord. 277, 55-64 (2020).

14. Tomova, L. et al. Nat. Neurosci. 23, 1597-1605 (2020).

Competing interests

The authors declare no competing interests. 